\title{
Rational AnTimicrobial Therapy in Infective Endocarditis
}

\section{Amirtha Ganesh *}

Antibiotic therapy has improved survival in IE by $70-80 \%$ and has been shown to reduce the incidence of complications of endocarditis. The fundamental goal of therapy-the eradication of the pathogen from the infected tissue-is made more difficult to achieve by specific and nonspecific defense mechanisms, e.g., biofilm formation and increasing tolerance or resistance to various antibiotics. If the patient's general condition is critical, then empirical, broad-spectrum antimicrobial therapy is begun as soon as blood cultures have been drawn. The early initiation of appropriate antibiotic therapy is very important, because it can not only control the local infection, but also lessen the risk of complications such as septic embolization.

Two basic principles for eradication of the infective organisms from the vegetations

1. Prolonged course of antibiotic treatment (4-6 weeks) is necessary to eradicate infection because bacterial concentration within vegetations is as high as 109 to $1011 \mathrm{CFU} / \mathrm{g}$ of tissue and organisms deep within vegetations are in accessible to phagocytic cells. Consenses dictates that counting of days for recommended duration of treatment should begin on the first day of negative blood cultures in cases where blood cultures had bben positive previously. For patients who undergo valve replacements for endocarditis, the post operative course of antibiotic should be similar to that prescribed for prosthetic valve endocarditis rather than the native valve endocarditis.

2. In parental administration of antibiotic therapy is necessary to achieve adequate drug levels required to eradicate infection. Parental therapy is typically initiated in the hospital setting, and the patient may be transitioned to outpatient parental antibiotic therapy for the remaining duration after an initial period of observation to assess for clinical response to therapy (at least two weeks).

A distinction should be made between native-valve endocarditis and prosthetic-valve endocarditis, which can, in turn, be either early (less than 1 year after valve replacement) or late (more than one year after valve replacement). A different spectrum of pathogens is to be expected in each of these cases. The spectrum of causative organisms in native-valve endocarditis and late prosthetic-valve endocarditis mainly consists of methicillin-sensitive $\mathrm{S}$. aureus strains, various streptococcal species, and Enterococcus faecalis. Early prosthetic-valve endocarditis, on the other hand, is often due to methicillin-resistant $\mathrm{S}$. aureus strains, coagulase-negative staphylococci, or gramnegative pathogens.

\section{CurRent Aspects Of ANTIBIOTIC Therapy}

Treatment should be started on an empirical basis, then modified once resistance data have been obtained. The choice of a suitable antibiotic should take the minimal inhibitory concentration (MIC) into account and not merely be based on a discrete classification of antibiotic sensitivity, e.g., into the three categories "sensitive," "intermediate," and "resistant." This holds particularly for treatment with glycopeptides: "vancomycin resistance," though often discussed, has been seen only rarely to date, including among S. aureus (VRSA), while the true knot of the problem lies in S. aureus strains that are vancomycin-intolerant, i.e., that have only intermediate sensitivity to vancomycin (VISA). In such cases, treatment can be appropriately guided only by the precise determination of resistances, along with adequate monitoring of serum trough levels; according to the current recommendations, these should lie in the range of $15-20 \mu \mathrm{g} / \mathrm{mL}$ and not, as previously recommended, $5-10 \mu \mathrm{g} / \mathrm{mL}$.

Although most previously published guidelines recommended combination therapy with beta-lactams and gentamicin, this recommendation must be viewed critically, particularly with regard to the treatment of staphylococcal infections. More recent meta-analyses show that combination therapy with gentamicin is not clinically superior to betalactam monotherapy; it leads, instead, to significantly elevated nephrotoxicity. In the recent guideline, combination

\footnotetext{
* Dr.Amirtha Ganesh, MD, DNB , Asst.Professor, Dept. of Cardiology, MGMCRI, Puducherry
} 
therapy with gentamicin is designated as optional for the treatment of staphylococcal infection. For the treatment of infective endocarditis due to methicillin-resistant streptococci, not just vancomycin, but also newer agents are currently being discussed. The lipopeptide daptomycin, for example, has been studied in a prospective, randomized trial in patients with right heart endocarditis and has been approved for this indication. Unlike vancomycin, daptomycin was at least as effective as the combination of a beta-lactam antibiotic and gentamicin in the treatment of methicillin-resistant staphylococcal infection.

While daptomycin has been found effective against secondary pulmonary abscesses caused by the embolization of infected vegetations, it is of no use against primary pulmonary infections, because the agent interacts with pulmonary surfactant. The nephrotoxicity of daptomycin was markedly less than that of comparable treatment with a combination of vancomycin, or a semisynthetic penicillin, with gentamicin. The oxazolidinone linezolide has also been used successfully in a number of cases of IE, but there are, as yet, no prospective data on this treatment.

\section{ProphyLAXIS - When?}

Over the past few decades the efficacy of antimicrobial prophylaxis in preventing IE has been questioned. Recently after an extensive review of all available literature and consensus of experts, the guidelines are updated. These guidelines have concluded that endocarditis was more likely to result from bacteremia associated with daily activities than with a dental procedure. Antibiotic prophylaxis even if $100 \%$ effective is estimated to prevent only an extremely small number of cases of infective endocarditis. These recommendations surmised that antibiotic prophylaxis should not be prescribed solely on the basis of an increased lifetime risk of endocarditis, but on the basis of cardiac conditions associated with highest risk of an adverse outcome from endocarditis. Conditions that warrant IE prophylaxis prior to dental procedures and procedures on respiratory tract, skin and musculoskeletal are

1. Patients with a prosthetic valve or a prosthetic material used for cardiac valve repair.

2. Patients with previous IE.

3. Patients with congenital heart disease

a. Cyanotic congenital heart disease, without surgical repair, or with residual defects, palliative shunts or conduits

b. Congenital heart disease with complete repair with prosthetic material whether placed by surgery or by percutaneous technique, up to 6 months after the procedure.

c. When a residual defect persists at the site of implantation of a prosthetic material or device by cardiac surgery or percutaneous technique.

Antibiotic prophylaxis is no longer recommended in other forms of valvular or congenital heart disease. IE prophylaxis before GI or genitourinary procedure is no longer recommended. 